\title{
FRENTE A LA CRISIS: EL COMPORTAMIENTO DE LAS PYMES QUERETANAS DURANTE LA CRISIS
}

\author{
Humberto Banda Ortiz* \\ Ignacio Almaraz Rodríguez
}

(Recibido: febrero 2014/Aceptado: junio 2014)

\section{Resumen}

Los periodos de las crisis financieras pueden afectar de muy diferentes maneras el funcionamiento de las pymes, llegando incluso a poner en riesgo su continuidad y su supervivencia. No obstante, no todas las pymes se ven igualmente afectadas por una crisis financiera. De hecho, algunas pymes pueden, incluso, prosperar y crecer durante las crisis, lo que propicia el incremento de sus utilidades. Existe evidencia empírica que sugiere que algunas pymes ven en las crisis financieras la oportunidad de desarrollar nuevas estrategias e innovar en los productos y/o servicios que ofrecen. Responder a las preguntas de ¿cuál fue el impacto de la crisis financiera de las hipotecas en las pymes queretanas?, y ¿cómo respondieron las pymes queretanas durante la crisis de las hipotecas?, son las dos cuestiones centrales que se abordan en el presente trabajo.

Palabras clave: crisis financieras, pymes.

Clasificación JEL: L22 L53

\footnotetext{
*Profesor-investigador de la Universidad Autónoma de Querétaro. <humberto.banda@gmail.com>.

** Profesor-investigador de la Universidad Autónoma de Querétaro <ignacioalmaraz@aol.com>.
} 


\section{Abstract}

Through financial crises periods, SMEs could view affected their operations in many different ways and, even more, SMEs could face the possibility to jeopardize their survival. However, not all SMEs are equally affected by a financial crisis. In fact, some SMEs may even thrive and grow during the crisis periods, which increased their profits. There are empirical evidence that suggest that some SMEs view in financial crises the opportunity to develop new strategies and innovate in their products and/or their services. Answer the following questions: what was the mortgage crisis on SMEs in Queretaro State?, and how SMEs in Queretaro State responded during the mortgage crisis?, is the central issue in this paper.

Keyworlds: financial crisis, SMEs.

JEL classification: L22, L53.

\section{Introducción}

Los acontecimientos que se presentaron en los mercados financieros internacionales en la segunda mitad del año 2007 pusieron de moda, nuevamente, el concepto de crisis financieras, término ampliamente mencionado en la década de los noventa del siglo pasado.

No obstante el fenómeno de las crisis financieras no es algo nuevo. A lo largo de los últimos siglos se han vivido crisis financieras en todo el mundo. En Estados Unidos las hubo en los siglos XIX y xx, y en Europa se remontan a una época tan antigua como la del auge y derrumbe del comercio de bulbos de tulipán en Holanda en el siglo xvir.

Durante la última década del siglo pasado se presentaron crisis financieras en diferentes países alrededor del mundo que combinaron elementos de fragilidad bancaria y fiscal, así como desequilibrios en los tipos de cambio de las diferentes divisas. Estas crisis financieras fueron el resultado de la salida de capitales de diversas economías como fue el caso de México 1994; Asia en 1997, Rusia 1998; Brasil 1999 y Argentina, 2001-2002, así como del contagio que tuvieron algunas economías emergentes.

En 2007, después de un periodo de relativa calma, las crisis financieras retornaron al mundo con la explosión de la burbuja inmobiliaria en Estados Unidos. Un año después, en septiembre de 2008, la quiebra del banco de 
inversión Lehman Brothers marcó el inicio de una crisis financiera a nivel global.

La crisis de las hipotecas se puso de manifiesto a partir de junio de 2007, no obstante desde 2006 muchos estudiosos de la materia ya mostraban signos de preocupación por los indicadores fundamentales que mostraban la economía de Estados Unidos.

La economía mexicana se mostró especialmente vulnerable ante las perturbaciones externas derivadas de la crisis de las hipotecas, propiciando que México tuviera una disminución del monto de sus exportaciones, una disminución en el monto de las remesas de los ciudadanos mexicanos que viven en Estados Unidos, una disminución de los puestos de trabajo, el aumento del tipo de cambio, el aumento de los requisitos para poder acceder a diferentes formas de financiamiento y el cierre de empresas, principalmente pymes.

El impacto de la crisis de las hipotecas en México motivó cambios en la política económica mexicana y también en la política social, lo que ocasionó una alta volatilidad de los indicadores económicos clave, como las tasas de consumo e inversión, el tipo de cambio real, la inflación y el producto interior bruto (PIB).

Por lo que respecta a las empresas, existe un limitado número de estudios que plantean que la crisis de las hipotecas obligó a éstas a modificar las estrategias que habían implementado respecto a la inversión, ahorro, exportación e importación, lo que afectó al comercio nacional e internacional, a la inversión extranjera directa y a los créditos. No obstante, durante el transcurso de esta investigación no se encontraron estudios que muestren cómo las pymes en el estado de Querétaro reaccionaron para hacer frente a la crisis de las hipotecas por lo cual cobra relevancia el presente trabajo.

El presente artículo se estructura de la siguiente manera: en la segunda sección se presentan la revisión de la literatura respecto al impacto que tienen las crisis financieras en las empresas. En la tercera sección se presenta la metodología empleada en el presente trabajo. En la cuarta sección se presentan los resultados obtenidos. Finalmente, en la quinta sección se presentan las conclusiones.

\section{Las empresas ante las crisis}

Las crisis financieras constituyen una fuente de riesgo tanto para las empresas como para los consumidores (Ang, Leong y Kotler, 2000). De acuerdo 
con Srinivasan, Rangaswamy y G. Lilien (2005), las crisis financieras pueden llegar a afectar el desempeño de la empresa e incluso poner en riesgo su continuidad y supervivencia. No obstante, en los trabajos de Kitching et al., (2009) y Meyer, Brooks y Goes (1990), se plantea que si bien las crisis representan amenazas para las organizaciones también son una fuente de oportunidades, por lo que las diferentes organizaciones deben de aprovechar los momentos de crisis para fortalecer su ventaja competitiva.

Estudios como los presentados por Domowitz, Hubbard y Pearson (1988) y Gabisch y Lorenz (1987) plantean que el impacto que tienen las crisis financieras es diferente en cada empresa y en cada región. Así mismo, dichos autores establecen en sus estudios que no todas las empresas presentan resultados pobres en sus operaciones o quiebran en los periodos de crisis, existen también empresas que prosperan y crecen durante dichos periodos.

Dobbs, Jesudason y Malige (2002) y Rigby (2001) establecen en sus trabajos que los directivos de algunas empresas, como por ejemplo los de General Motors, Microsoft y Wal-Mart, ven en los periodos de crisis la oportunidad para fortalecer a sus empresas, incrementar sus niveles de inversiones y desplazar a sus competidores más débiles. Sin embargo, es normal que ante una crisis financiera las empresas disminuyan su nivel de gastos y pospongan nuevas inversiones, lo que para Quelch \& Jocz (2009) y Burgers (2009) representa un error.

Las empresas presentan una amplia variedad de respuestas ante las crisis financieras, lo que provoca que el desempeño de las empresas sea incierto durante estos periodos (Wan y Yiu, 2009). Así mismo, Kunc y Bhandari (2011) plantean que algunas organizaciones ponen en riesgo su continuidad y supervivencia al descuidar aspectos estratégicos como son la satisfacción de sus clientes y la innovación en sus procesos y servicios.

Como se planteó en el párrafo anterior, no existe un consenso sobre cual debería de ser la respuesta de las empresas ante una crisis financiera, sin embargo, Gunby (2009) sugiere que en épocas de crisis existe un compromiso por parte de los empresarios para desarrollar estrategias que permitan responder exitosamente ante los riesgos del entorno. En este mismo sentido, Navarro, Bromiley y Sottile (2010) argumentan que los directivos de las diferentes organizaciones pueden prepararse para hacer frente a las crisis financieras estableciendo a priori estrategias adecuadas, como por ejemplo, innovaciones e inversiones anticíclicas. 
Siguiendo a Chattopadhyay, Glick y Huber (2001), se puede expresar que la decisión entre innovar e invertir, o reducir los niveles de gasto de la empresa en los periodos de crisis debe ser impulsada por los directivos de las organizaciones, los cuales tendrán que convencer a los accionistas de su decisión. Así mismo, se debe evaluar el riesgo que representa invertir durante un periodo de crisis contra el riesgo que se tiene de perder ventajas competitivas por no invertir. Cabe señalar que existen diferentes trabajos que documentan que las empresas pueden obtener ventajas competitivas en los periodos de crisis financieras si invierten en el desarrollo de nuevos productos e innovación (Rhodes y Stelter, 2009).

Durante el periodo de crisis, por lo general, el consumo de los clientes tiende a la compra de productos más económicos para ahorrar, lo que provoca un incremento en las ventas de las empresas que producen dichos bienes y/o servicios y contrae los ingresos de las empresas con altos precios. Así mismo, de acuerdo con lo planteado por Rosier (2011), las crisis financieras tienen como consecuencias cambios en la demanda de los clientes tanto en volumen como en preferencias, por lo que aquellas empresas que estén orientadas al mercado y sean ágiles para adecuar sus procesos, de tal modo que puedan satisfacer las nuevas necesidades de sus clientes, pueden crear valor para la organización.

Por lo que respecta a las pymes, Smallbone et al. (2012), establecen factores tales como el tamaño de la empresa y el sector en donde se desenvuelve tienden a influir en la forma en que las organizaciones se adaptan y se desempeñan durante un periodo de crisis.

En sus investigaciones Geroski y Gregg (1997) y Latham (2009), demuestran de manera empírica que, normalmente, las pymes presentan una mayor vulnerabilidad ante una crisis financiera. No obstante, de acuerdo con Kalantaridis (2004), no existe un consenso en la literatura sobre las ventajas y desventajas que tienen las empresas por su tamaño.

Para Curran, West y Finch (1996) el tamaño de la empresa es importante en un momento de crisis porque puede llegar a afectar la capacidad que tiene para responder a los cambios en el mercado. Este mismo autor plantea que de acuerdo con el tamaño de las empresas son diferentes los mecanismos por los que se ven afectadas en los momentos de crisis y nivel de impacto.

En un trabajo presentado por Shama (1993) se establece que las empresas pequeñas y grandes tienen diferentes percepciones de los efectos que 
tienen las crisis financieras, lo que provoca que reaccionen de diferente manera cuando tienen que hacer frente a una crisis. Para dicho autor, esto se debe a que las empresas de diferentes tamaños enfrentan diversos entornos económicos, debido a que atienden a diferentes nichos de mercado y a la participación de mercado que poseen.

De acuerdo con Shama (1993) las pymes se enfrentan a dos escenarios, el primero es tener un nicho de mercado protegido que le ayude a sortear la crisis, el segundo es que no puedan acceder a las diferentes fuentes de financiamiento para sostener sus operaciones y quiebren. Para este autor, el segundo escenario es el más común, por ello existe un mayor número de pymes que suspenden operaciones en los periodos de crisis que de empresas grandes.

Shama (1993), Beaver y Ross (2000), expresan lo contrario, ellos plantean que las pymes pueden prosperar durante los periodos de crisis si poseen una estructura idónea de capital a largo plazo, tienen cercanía con sus clientes y plantean una estrategia proactiva. En este mismo sentido, DeDee y Vorhies (1998) exponen que las pymes podrían obtener una ventaja competitiva si poseen la capacidad de adaptarse al entorno, lo que debería ser lo normal; ya que regularmente en las pymes existe un menor nivel de burocracia que en las grandes empresas, lo que propicia que puedan tomar decisiones de manera ágil y rápida debido a su flexibilidad estructural.

Otro elemento que hay que considerar, respecto al tamaño de las empresas, son las economías de escala que se pueden llegar a producir en los periodos de crisis. Serrasqueiro y Maçãs (2008) establecen que es más factible que las grandes empresas aprovechen las economías de escala que las pymes. Además, mientras más grande sea la empresa existe una mayor posibilidad de que tenga diversificados sus productos, lo que permitirá hacer frente a los posibles cambios en el comportamiento de sus clientes. No obstante, cabe señalar que la teoría económica plantea que existen rendimientos marginales decrecientes cuando se sobrepasa el tamaño óptimo de la empresa.

Como se puede apreciar por lo planteado en los párrafos anteriores de esta sección no existe un consenso entre los diferentes autores y estudios sobre la forma en la cual se desempeñan las pymes en los momentos de crisis, es por ello que cobra relevancia saber ¿cuál fue el impacto de la crisis de las hipotecas en las pymes queretanas?, y ¿cómo respondieron las pymes queretanas durante la crisis de las hipotecas?, en la siguiente sección se presenta la metodología que se empleó para contestar estas preguntas. 


\section{Metodología}

En esta sección se describe la metodología empleada para contestar a las dos preguntas en las que se fundamenta la presente investigación.

El enfoque metodológico empleado en el presente trabajo fue de tipo no experimental descriptivo. Se utilizó la modalidad de encuesta de diseño transversal o transeccional, ya que fue llevada a cabo en un periodo temporal concreto. La herramienta que se usó para la realización de la encuesta fue un cuestionario cuantitativo con rasgos cualitativos, que tenía como objetivo recolectar la información necesaria para contestar las preguntas de investigación.

La utilización de cuestionarios permitió: desarrollar un procedimiento mediante el cual se obtuvieron datos que fueron analizados mediante técnicas estadísticas (perspectiva cuantitativa), y comprender mejor el significado que tienen algunos conceptos para los encuestados (perspectiva cualitativa).

La elaboración del diseño metodológico y puesta en marcha de la investigación siguió un procedimiento estructurado en seis etapas que se describen en la tabla 1.

Tabla 1

Etapas en el proceso de investigación

\begin{tabular}{|c|c|c|}
\hline Fase & \multicolumn{1}{c}{ Procedimiento } & \multicolumn{1}{c|}{ Secuencia } \\
\hline 1 & $\begin{array}{c}\text { Revisión } \\
\text { bibliográfica }\end{array}$ & $\begin{array}{c}\text { enero 2010 a julio } \\
2013\end{array}$ \\
\hline 2 & $\begin{array}{c}\text { Elaboración } \\
\text { de cuestionario }\end{array}$ & enero a junio 2011 \\
\hline 3 & $\begin{array}{c}\text { Validación } \\
\text { del cuestionario } \\
\text { Aplicación } \\
\text { de cuestionarios }\end{array}$ & julio a diciembre 2011 \\
\hline 5 & $\begin{array}{c}\text { procesamiento y } \\
\text { análisis de datos }\end{array}$ & $\begin{array}{c}\text { junio a diciembre } \\
2012\end{array}$ \\
\hline 6 & $\begin{array}{c}\text { redacción del } \\
\text { informe }\end{array}$ & enero a mayo 2013 \\
\hline
\end{tabular}

Fuente: elaboración propia. 
De acuerdo con las preguntas objeto de estudio del presente trabajo se definió la población que sería objeto de estudio. El criterio que se estableció para la inclusión de los participantes en la muestra fueron las empresas que de acuerdo al número de trabajadores que emplean son consideradas en la literatura tradicional como pymes y que, adicionalmente, tengan la base de sus operación en el estado de Querétaro. Cabe señalar que durante el transcurso de la presente investigación no fue factible encontrar datos fiables del número de pymes que operan en el estado de Querétaro por lo que no fue factible determinar el tamaño de la población.

Por lo expresado en el párrafo anterior, para la aplicación de la encuesta se recurrió a localizar a los posibles participantes en el estudio mediante la búsqueda en la web y el registro en las diferentes cámaras de las empresas susceptibles a ser encuestadas. Este esfuerzo condujo a la aplicación de cuarenta y dos encuestas. Las características de las empresas encuestadas se presentan en la tabla 2.

Tabla 2

Características de la muestra

\begin{tabular}{|l|c|c|l|c|c|}
\hline \multicolumn{2}{|c|}{ Tamaño } & \multicolumn{3}{c|}{ Giro } \\
\hline \multicolumn{2}{|c|}{ número } & $\%$ & \multicolumn{2}{c|}{ número } & $\%$ \\
\hline $\begin{array}{l}\text { Micro } \\
(<10 \text { empleados })\end{array}$ & 30 & $71.43 \%$ & Manufatura & 14 & $33.33 \%$ \\
\hline $\begin{array}{l}\text { Pequeñas } \\
(10-49 \text { empleados })\end{array}$ & 11 & $26.19 \%$ & Construcción & 12 & $28.57 \%$ \\
\hline $\begin{array}{l}\text { Medianas } \\
(50-249 \text { empleados })\end{array}$ & 1 & $2.38 \%$ & Servicios & 1 & $2.38 \%$ \\
\hline & & & Ventas & 10 & $23.81 \%$ \\
\hline Total & 42 & $100.00 \%$ & Total & 42 & $100.00 \%$ \\
\hline
\end{tabular}

Fuente: elaboración propia.

Una vez establecida la metodología que se utilizó para la recolección de los datos necesarios para alcanzar los objetivos que se plantearon en la presente investigación en la siguiente sección se presentan los resultados obtenidos. 


\section{Resultados}

En esta sección se exponen los resultados del análisis descriptivo elaborado a partir de los datos que se obtuvieron en la aplicación de los cuestionarios. Con la finalidad de hacer más clara la exposición, los resultados se presentarán de acuerdo con las preguntas de investigación que se plantearon al inicio del presente trabajo.

Por lo que respecta al impacto que tuvo la crisis de las hipotecas en las operaciones normales de las pymes en el estado de Querétaro, únicamente el $21.4 \%$ de los encuestados consideró que fue seriamente afectado por la crisis económica. El mayor porcentaje, un 42.9\%, consideró que fue moderado el impacto de la recesión. Cabe señalar que un $14.21 \%$ de las personas encuetadas manifestó que no tuvo ningún impacto (figura 1).

\section{Figura 1}

Impacto de la recesión en las empresas encuestadas

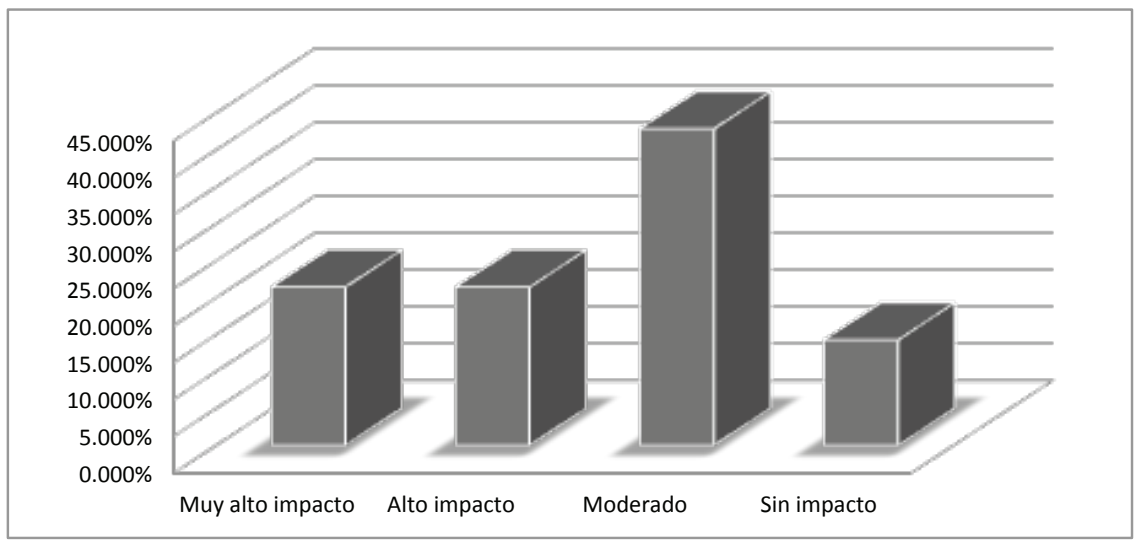

Fuente: elaboración propia.

Durante la investigación se pudo constatar que las pymes del estado de Querétaro perdieron competitividad durante el periodo que duró la crisis de las hipotecas. De los encuestados un 58.72\% planteo que su empresa perdió competitividad frente a otras empresas durante la crisis, mientras que un $41.28 \%$ no vieron afectada su competitividad (figura 2). 
Figura 2

Pérdida de competitividad durante la crisis

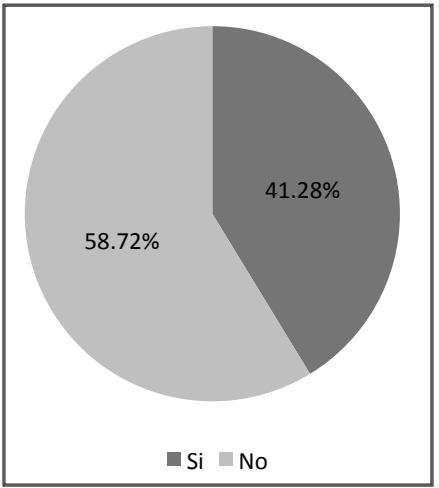

Fuente: elaboración propia.

Así mismo, un 33\% de las pymes encuestadas disminuyeron su nivel de ingresos debido a la disminución en la demanda de sus producto, un 27.78 $\%$ por el incremento de precios de sus productos y un $23.33 \%$ por el incremento en los costos de los materiales que ocupan (figura 3).

\section{Figura 3}

Causas de la pérdida de ingresos

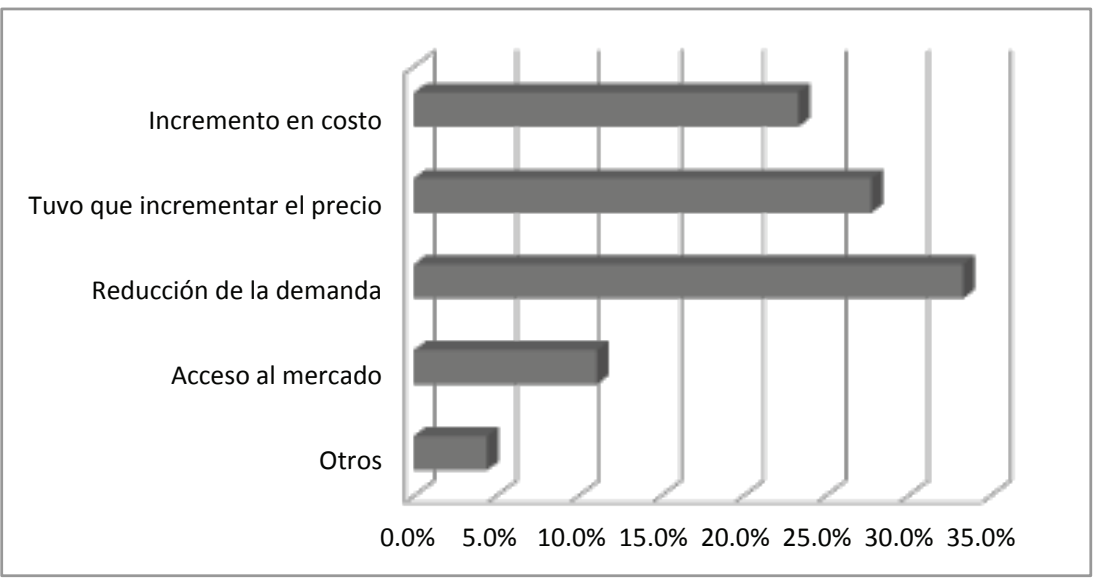

Fuente: elaboración propia. 
En cuanto al tiempo en el los directivos de las empresas encuestadas sintieron el impacto de la crisis de las hipotecas en sus empresas, un 69\% manifestó que ésta se vio reflejada en sus operaciones en menos de seis meses, mientras que el 32\% restante consideró que sus empresas se vieron afectadas sus operaciones en un periodo de seis meses a un año (figura 4).

\section{Figura 4}

Tiempo que tardó en impactar la crisis en las operaciones

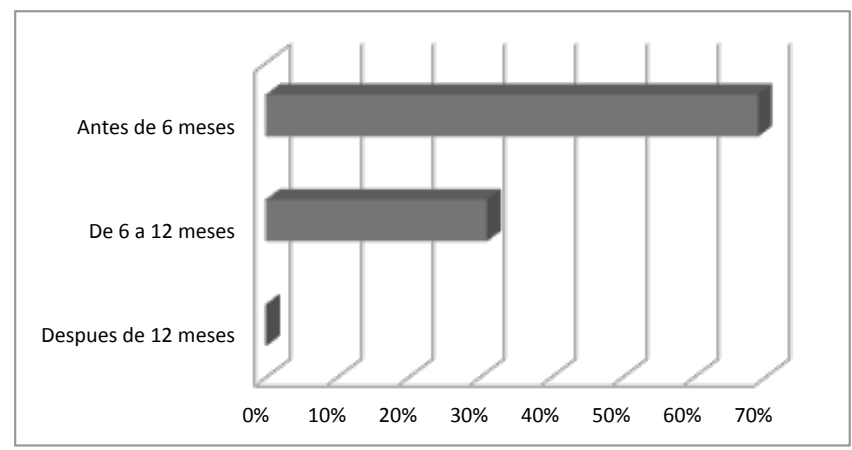

Fuente: elaboración propia.

No obstante los efectos que la crisis de las hipotecas tuvo tanto en las operaciones como en los ingresos de las pymes queretanas sólo un 16.67\% de los empresarios encuestados manifestaron que existiera el riesgo de que su empresa suspenda sus operaciones o quiebre (figura 5).

\section{Figura 5}

Existe el riesgo de que su empresa cierre por causa de la crisis

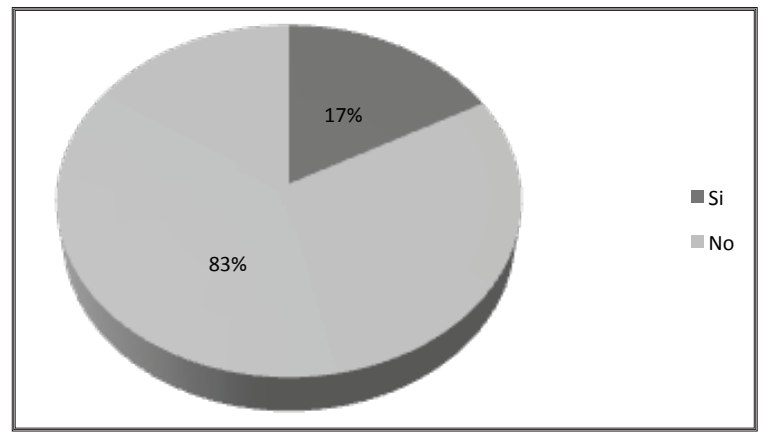

Fuente: elaboración propia. 
Para hacer frente a la crisis los directivos de las pymes modificaron sus estrategias de muy diferentes manera. La acción principal que tomaron los directivos de las pymes encuestadas fue la de buscar nuevos mercados en los cuales vender sus productos y/o servicios, un $90.48 \%$, seguida de un incremento en el esfuerzo de ventas en los mercados actuales, un $83.33 \%$, y la disminución en el número de empleados que contrataban, un 80.95\%, (figura 6).

Figura 6

Acciones tomadas por las pymes en el periodo de crisis

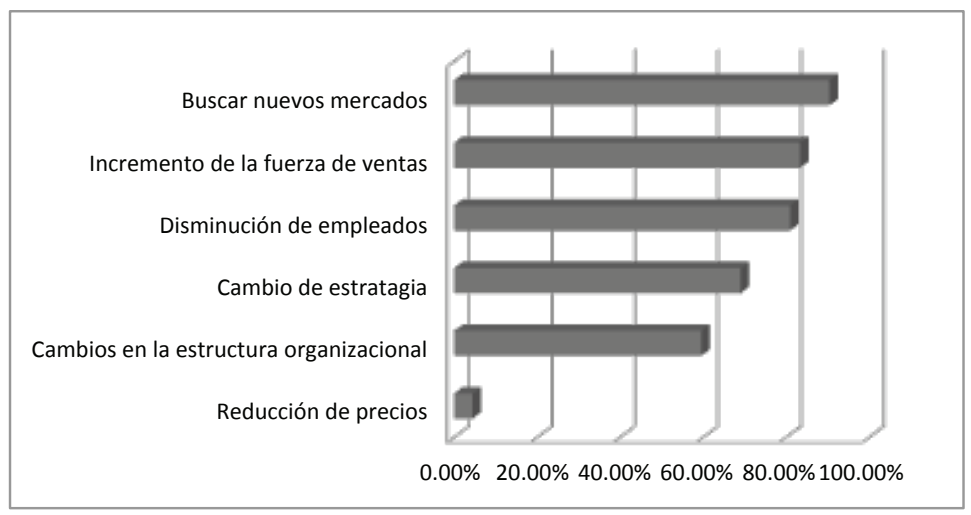

Fuente: elaboración propia.

De las empresas encuestadas un $97.62 \%$ manifestaron que se vieron obligadas a suspender sus inversiones. La reducción de las inversiones de las pymes en el estado de Querétaro se vio reflejada, principalmente, en la compra de maquinaria y equipo, un $97.62 \%$, y en la capacitación que se brindaba al personal, un $95.24 \%$. Adicionalmente, cabe señalar que las pymes queretanas se mostraron especialmente cautelosas al momento de recurrir al financiamiento externo durante la crisis de las hipotecas, sólo un $7.14 \%$ incrementaron sus niveles de deuda (figura 7). 


\section{Figura 7}

Acciones tomadas por las pymes en el periodo de crisis

(Aspectos financieros)

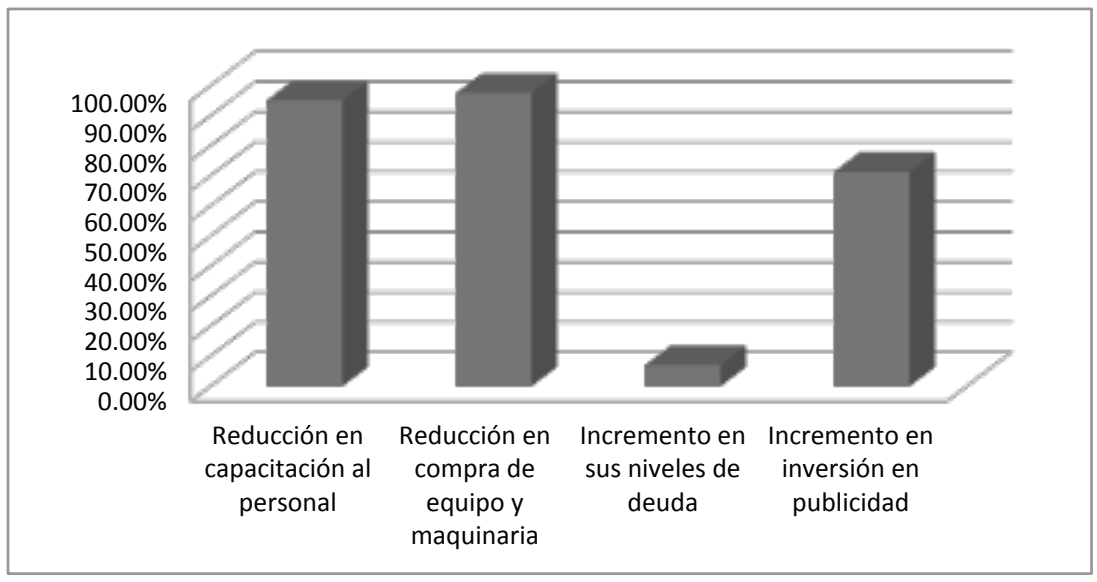

Fuente: elaboración propia.

\section{Conclusiones}

El objetivo del presente estudio fue determinar cómo influyó la crisis financiera de las hipotecas en las pymes del estado de Querétaro y la forma en que dichas empresas respondieron. La crisis de las hipotecas planteó, nuevamente, un escenario de incertidumbre repentino en el estado, que afectó a las empresas y a los consumidores.

Comúnmente, en el ambiente empresarial se menciona que la crisis de las hipotecas tuvo consecuencias negativas en las empresas en general y sobre las pymes en particular, a tal grado que muchas tuvieron que suspender parte de sus operaciones o quebraron. Si bien dichas aseveraciones son indiscutibles, durante la revisión de la literatura se encontraron diversos estudios que plantean que las crisis son momentos de oportunidades para ciertas empresas, por lo que el reto que tienen los directivos es aprovechar las oportunidades que generan la crisis para obtener ventajas para su organización.

El diseño de la presente investigación permitió ofrecer una visión más precisa de cómo las crisis de las hipotecas influyerón en las actividades y en el desempeño de las pymes en el estado de Querétaro. 
Durante la investigación se encontró que la crisis impactó a las pymes queretanas en una amplia variedad de formas, de tal forma que algunas percibieron un muy alto impacto, un alto impacto, un impacto moderado e incluso hubo pymes que no registraron ningún impacto, por lo que se debe de rechazar el argumento de que las crisis impactan, necesariamente, de una forma negativa a las pymes.

La mayoría de las pymes en Querétaro manifestaron que perdieron competitividad durante la crisis. Además, la mayoría de estas empresas vieron afectados sus ingresos principalmente por la reducción en su volumen de ventas y por el incremento en el costo de sus insumos.

Adicionalmente, la forma en cómo los directivos de las pymes queretanas se adaptaron a la crisis de las hipotecas fue diversa, de tal forma que más de las tres cuartas partes de ellos manifestaron que no existía la posibilidad de que quebraran o suspendieran sus operaciones totalmente.

Los directivos de las pymes en Querétaro implementaron diversas estrategias en sus empresas para sortear de mejor forma la crisis de las hipotecas. Entre dichas estrategias sobresalen la búsqueda de nuevos nichos de mercado, el incremento de la labor de ventas, la reducción de personal y el cambio de estrategia.

Cabe señalar que en la revisión de la literatura se encontró que algunas empresas pueden encontrar en la crisis la oportunidad de expandirse mediante la inversión, la innovación y la diversificación; no obstante la mayoría de las pymes queretanas optaron por contraer sus niveles de apalancamiento, reducir sus inversiones en activos fijos y disminuir sus costos de capacitación al personal.

Finalmente cabe resaltar que la crisis de las hipotecas planteó tanto una amenaza como una oportunidad para las pymes en el estado de Querétaro. Si bien es cierto que dichas empresas tienen recursos limitados, y que esto las hacen más sensibles a los efectos de las crisis, también es cierto que mediante las estrategias que implementaron pudieron sortear el periodo de crisis de forma favorable, por lo que la vulnerabilidad que presentan las empresas ante las crisis no es una característica que se pueda atribuir a las pymes en el estado de Querétaro, ya que a través de las estrategias que implementen sus directivos fueron capaces de influir de manera importante en su desempeño y, por ende, en su supervivencia. 


\section{Bibliografía}

Ang, S. (2001). "Crisis marketing: a comparison across economic scenarios", International Business Review, vol, 10, pp. 263-284.

Ang, S.H.; S.M. Leong y P. Kotler (2000). “The Asian apocalypse: crisis marketing for consumer and businesses", Long Range Planning, vol. 33, pp. 97-119.

Beaver, G. y C. Ross (2000).“Enterprise in Recession: The Role and Context of Strategy", International Journal of Entrepreneurship and Innovation, 1 (1), pp. 23-31

Burgers, W. (2009). "Marketing during economic downturns", Financial Times, Managing in a Downturn Series, February 5.

Chattopadhyay, P.; W. H. Glick y G. P. Huber (2001). “Organizational actions in response to threats and opportunities". Academy of Management Journal, 44 (5), pp. 937-955.

Curran, P. J.; S. G. West y G. F. Finch (1996). "The robustness of test statistics to nonnormality and specification error in confirmatory factor analysis". Psychological Methods, 1, pp. 16-29.

DeDee, K.J. y D.W. Vorhies (1998). Retrenchment activities of small firms during economic downturn: An empirical investigation. Journal of Small Business Management, 36 (3), pp. 46-61.

Dobbs, R.F.; D. Robert Jesudason and Francis H. Malige (2002). "Moving up in a Downturn", McKinsey on Finance, 9-14.

Domowitz, I.; R.G. Hubbard y B.C. Peterson (1988). "Market Structure and Cyclical Fluctuations in U.S. Manufacturing". The Review of Economics and Statistics, 70 (1), pp. 55-66.

Gabisch, G. y H. Lorenz (1987). Business Cycle Theory: A Survey of Method and Concepts. New York, NY: Springer-Verlag.

Geroski, P.A. y P. Gregg (1994). "Corporate restructuring in the UK during the recession". Business Strategy Review, 5 (2), pp. 1-19.

Gunby, N. W. Jr. (2009). "Firm performance and complementary strategy development processes". Management Decision, 47 (5), pp. 806-818.

Hartman, N. (2009). "Sure ways to tackle uncertainty in tough times", Financial Times, Managing in a Downturn Series, February 5,

Kitching, J.; R. Blackburn; D. Smallbone y S. Dixon (2009). Business strategies and performance during difficult economic conditions. Department of Business Innovation and Skills (BIS).

Kunc, M. y R. Bhandari (2011). "Strategic development processes during economic and financial crisis". Management Decision, 49 (8), pp. 1343-1353. 
Latham, S. (2009). "Contrasting strategic response to economic recession in start-up versus established software firms". Journal of Small Business Management, 47 (2), pp. 180-201.

Meyer, A.; G.,Brooks y J. Goes (1990). “Environmental jolts and industry revolutions: organizational responses to discontinuous change", Strategic Management Journal, vol. 11, núm. 5, pp. 93-110.

Navarro, P.; P. Bromiley y P. Sottile (2010). "Business cycle management and firm performance: Tying the empirical knot". Journal of Strategy and Management, 3 (1), pp. 50-71.

Quelch, J.; K. Jocz (2009). "How to Market in a downturn”, Harvard Business Review, vol. 87, núm. 4, pp. 52-62.

Rigby, D. (2001). "Moving Upward in a Downturn," Harvard Business Review, 79 (July), pp. 99-105.

Rhodes, D. y D. Stelter (2009). "Seize advantage in a downturn". Harvard Business Review, 87 (2), pp. 49-59.

Rosier, E. (2011). "Marketing strategy in a turbulent environment". Journal of StrategicMarketing, 19 (5), pp. 413-419.

Shama, A. (1993). "Marketing strategies during recession: A comparison of small and large firms". Journal of Small Business Management, 31 (3), pp. 62-72.

Smallbone, D.; D. Deakins, M. Battisti, y J. Kitching (2012). “Small business responses to a major economic downturn: Empirical perspectives from New Zealand and the United Kingdom". International Small Business Journal, 0 (0) pp. 1-24.

Srinivasan, R.; A. Rangaswamy y G. Lilien (2005). “Turning adversity into advantage: does proactive marketing during a recession pay off?", International Journal of Research in Marketing, vol. 22, núm. 2, pp. 109-125.

Wan, W. P.; D. W. Yiu (2009). “From crisis to opportunity: Environmental jolt, corporate acquisitions, and firm performance". Strategic Management Journal, 30 (7), pp. 791-801.

Serrasqueiro, Z. y P. Maçãs (2008). "Performance and size: empirical evidence from Portuguese SMEs," Small Business Economics, Springer, vol. 31 (2), pp. 195-217. 